\title{
DIFFERENTIABILITY ALMOST EVERYWHERE
}

\section{J. NEUGEBAUER ${ }^{1}$}

1. In this paper we will give necessary and sufficient conditions for a measurable function to be equivalent to one which is differentiable a. e. on a set $E$. The condition is in terms of Marcinkiewicz type integrals which have also been recently the main objects in problems of differentiability. The reader is especially referred to the important paper on differentiability by E. M. Stein and A. Zygmund [8]. Their results are crucial for our paper; in fact, our paper is essentially only a slight refinement of their results and of a theorem due to Marcinkiewicz [2].

Let $I_{0}=[0,1]$, and let $f: I_{0} \rightarrow R$ be a measurable function, where $R$ is the set of real numbers. We will abbreviate the second symmetric difference of $f$ at $x$ by $\Delta^{2} f(x, t)$, i.e., $\Delta^{2} f(x, t)=f(x+t)+f(x-t)-2 f(x)$. It will also be useful to retain the notation $\epsilon_{x}(t)=\left|\Delta^{2} f(x, t)\right|(2 t)^{-1}$ introduced in [8]. We say that two functions are equivalent if they differ on a set of measure zero. The measure of a set $A$ will be denoted by $|A|$. The function $\phi: R \rightarrow R$ is defined by $\phi(x)=1-|x|$, if $|x|<1$, and $\phi(x)=0,|x| \geqq 1$. We are now ready to state the main theorem of our paper.

Theorem 1. A measurable function $f: I_{0} \rightarrow R$ is equivalent to one which is differentiable a. e. on a measurable set $E \subset I_{0}$ if and only if for almost every $x \in E$ there is $\eta=\eta_{x}>0$ such that

$$
\int_{0}^{\eta} \frac{\epsilon_{x}^{2}(t)}{t \phi\left(\epsilon_{x}(t)\right)} d t<\infty .
$$

We remark that (1) appears as a limiting case of

$$
\int_{0}^{n} \frac{\epsilon_{x}^{2}(t)}{t} \frac{1-\epsilon_{x}^{n-1}(t)}{1-\epsilon_{x}(t)} d t .
$$

It has been shown by E. M. Stein and A. Zygmund [8, p. 280] that the finiteness of this integral a. e. in $E$ is equivalent with the existence of the $L_{n}$-derivative a. e. in $E$.

It is an immediate consequence of a theorem due to Marcinkiewicz [2] that if $f$ is differentiable a. e. on $E$, then for almost every $x \in E$ there is $\eta=\eta_{x}>0$ such that the sequence

Received by the editors December 22, 1964.

1 Supported by NSF Grant GP-1665. 


$$
\int_{0}^{\eta} \frac{\left|\Delta^{2} f(x, t)\right|^{p}}{t^{p+1}} d t, \quad p=2,3, \cdots,
$$

is bounded. We will show that (2) is also sufficient for $f$ to be equivalent to a function differentiable a. e. in $E$. By examples we will show that the "boundedness" cannot be relaxed.

2. We will collect now some definitions and lemmas which will prove to be useful. Let $f: I_{0} \rightarrow R$ be measurable, and let for $y \in R$, $E_{y}=\{x: f(x)>y\}$. For $x_{0} \in I_{0}$ define

$$
g\left(x_{0}\right)=\inf \left\{y:\left|E_{y} \cap I\right|=o(|I|), \text { as }|I| \rightarrow 0, x_{0} \in I\right\},
$$

where $I$ is the generic notation for an interval. The function $g: I_{0} \rightarrow R$, which can be defined for arbitrary functions by replacing "measure" by "outer measure," was introduced in [1] and is called the upper (measurable) boundary of $f$. The function $g$ has the following immediate properties.

( $\left.\mathrm{P}_{1}\right) f(x)=g(x)$ at every point $x$ of approximate continuity of $f$, and hence $f(x)=g(x)$ a. e.

$\left(\mathrm{P}_{2}\right)$ For each $x_{0} \in I_{0}$ and each $\epsilon>0, \eta>0$, the set $\left\{x:\left|x-x_{0}\right|<\eta\right.$ and $\left.\left|g(x)-g\left(x_{0}\right)\right|<\epsilon\right\}$ has positive measure.

If $f: I_{0} \rightarrow R$ is a measurable function, we will denote by $f_{*}^{\prime}(x)$ the derivative of $f$ at $x$ neglecting sets of measure zero, i.e., there is a set $N_{x},\left|N_{x}\right|=0$, such that $f_{*}^{\prime}(x)=\lim _{z \rightarrow x}[f(z)-f(x)](z-x)^{-1}, z \notin N_{x}$.

LEMma 1. Let $f: I_{0} \rightarrow R$ be measurable, and assume that $f_{*}^{\prime}(x)$ exists $x \in E$. Then $f$ is equivalent to a function which is differentiable on $E$.

Proof. Let $g$ be the upper boundary of $f$. Then $g(x)=f(x)$ a. e.; and, in particular, the equality holds for $x \in E$. Let $x_{0} \in E$ and let $\epsilon>0$ be given. There exists $\delta>0$ and a set $N,|N|=0$, such that $N \subset\left(x_{0}-\delta, x_{0}+\delta\right)$ and

$$
\left|\frac{g(z)-g\left(x_{0}\right)}{z-x_{0}}-f_{*}^{\prime}(x)\right| \leqq \epsilon,\left|z-x_{0}\right| \leqq \delta, \quad z \notin N .
$$

We will show that $N=\varnothing$. If $N \neq \varnothing$, the property $\left(\mathrm{P}_{2}\right)$ would imply that the inequality

$$
\left|\frac{g(z)-g\left(x_{0}\right)}{z-x_{0}}-f_{*}^{\prime}\left(x_{0}\right)\right|>\epsilon
$$

holds on a set of positive measure in $\left(x_{0}-\delta, x_{0}+\delta\right)$.

Lemma 2. Let $f: I_{0} \rightarrow R$ be measurable, and let $E \subset I_{0}$. Assume that for each $x \in E$ there is a set $N_{x}$ such that (i) $\left|N_{x}\right|=0$, (ii) $\Delta^{2} f(x, t)=O(t)$ 
as $t \rightarrow 0, t \notin N_{x}$. Then there exists a set $N \subset I_{0}$ such that $|N|=0$ and $\Delta^{2} f(x, t)=O(t), x \pm t \notin N$, for each $x \in E$.

Proof. Let $N$ be the set of all points in $I_{0}$ at which $f$ is not approximately continuous. Then $|N|=0$, and the desired property readily follows.

REMARK. It is important to note that the set $N$ above is independent of $x \in E$.

3. Let $f: I_{0} \rightarrow R$ be measurable and let $E \subset I_{0}$ be measurable. It is known [3] that the condition $\Delta^{2} f(x, t)=O(t), x \in E$, implies that the set of points in $E$ at which $f_{a p}^{\prime}$ (see, e.g., [6, p. 218]) exists has the same measure as the set of points in $E$ at which $f^{\prime}$ exists. We need a slight variant of this theorem obtained by relaxing the condition $O(t)$ to $O(t), t \notin N_{x},\left|N_{x}\right|=0$. In the conclusion, of course, $f^{\prime}$ is replaced by $f_{*}^{\prime}$. The proof proceeds along the lines of [3]. The measurability of certain sets will be established in much the same way as in [4] which contains an extension of the above mentioned result in [3] to several variables. Since the result is important for our purposes, we will give the essential steps of the proof.

LEMmA 3. Let $f: I_{0} \rightarrow R$ be measurable and let $E \subset I_{0}$ be measurable. Assume that for each $x \in E$ there is a set $N_{x}$ such that (i) $\left|N_{x}\right|=0$, (ii) $\Delta^{2} f(x, t)=O(t)$ as $t \rightarrow 0, t \notin N_{x}$. If $f_{a p}^{\prime}(x)$ exists for $x \in E$, then $f_{a p}^{\prime}(x)=f_{*}^{\prime}(x)$ a. e. in $E$.

Proof. By Lemma 2 there is a set $N \subset I_{0}$ such that $|N|=0$ and $\Delta^{2} f(x, t)=O(t), x \pm t \notin N$, for each $x \in E$. Let $F_{1} \subset F_{2} \subset \cdots$ be a sequence of closed sets in $I_{0}-N$ such that $f \mid F_{i}$ is continuous and $H=U F_{i}$ has measure $\left|I_{0}\right|=1$. Let us set $E(k, j)=\{x \in H \cap E$ : $\left.\left|\Delta^{2} f(x, t)\right|<k \cdot t, 0<t \leqq 1 / j, x \pm t \in H\right\}$. Then, as in [4], $E(k, j)$ is measurable and $H \cap E=U U E(k, j)$. Thus the relation $f_{a p}^{\prime}(x)=f_{*}^{\prime}(x)$ need only be verified at every point of density of $E(k, j)$. We may assume that 0 is such a point and that $f_{a p}^{\prime}(0)=0$. Let $\epsilon>0$ be given. There is a measurable set $E \subset E(k, j)$ such that 0 is a point of density of $E$ and

$$
|f(x)-f(0)|<(\epsilon / k)|x|, \quad x \in E .
$$

Let us now assume that $x>0$. The set $A_{x}=\left\{x^{\prime}:\left(x^{\prime}+x\right) / 2 \in E\right.$, $0<x^{\prime}<x$, and $\left.\left|x^{\prime}-x\right|<\epsilon x\right\}$ has measure

$$
\int_{(1-\epsilon) x}^{x} \chi\left(\frac{x^{\prime}+x}{2}\right) d x^{\prime} \simeq \epsilon x,
$$


where $\chi(u)$ is the characteristic function of $E$. Hence there is $0<\delta<1 / j$ such that $A_{x} \cap E \neq \varnothing, 0<x<\delta$. Thus, if $0<x<\delta$ and $x \in H$, then there is $x^{\prime} \in A_{x} \cap E$ and

$$
\begin{aligned}
|f(x)-f(0)| \leqq & \left|2 f\left(\frac{x^{\prime}+x}{2}\right)-f\left(x^{\prime}\right)-f(x)\right| \\
& +2\left|f\left(\frac{x^{\prime}+x}{2}\right)-f(0)\right|+\left|f^{\prime}\left(x^{\prime}\right)-f(0)\right|<5 \epsilon x .
\end{aligned}
$$

Hence $f_{*}^{\prime}(0)=0$, and the proof is complete.

4. We are now ready to prove Theorem 1 . Let us first assume that there exists a function $g: I_{0} \rightarrow R$ equivalent with $f$ such that $g^{\prime}(x)$ exists a. e. in $E$. By [2], for almost every $x \in E$ there is $\sigma=\sigma_{x}>0$ such that

$$
\int_{0}^{\sigma} \frac{\left|\Delta^{2} f(x, t)\right|^{2}}{t^{3}} d t<\infty .
$$

We will show that the integral (1) is finite at every $x$ at which $g^{\prime}(x)$ exists, $g(x)=f(x)$, and (3) holds. Since $\Delta^{2} g(x, t)=o(t)$, and $f(x)=g(x)$ a. e., there is $0<\eta<\sigma$, and $N_{x},\left|N_{x}\right|=0$, such that $\left|\Delta^{2} f(x, t)\right|<t, 0<t \leqq \eta, t \notin N_{x}$. Thus for $n \geqq 2$,

$$
\int_{0}^{n} \frac{\left|\Delta^{2} f(x, t)\right|^{n}}{2^{n} t^{n+1}} d t \leqq \frac{1}{2^{n}} \int_{0}^{n} \frac{\left|\Delta^{2} f(x, t)\right|^{2}}{t^{3}} a t
$$

from which we infer that

$$
\sum_{n=2}^{\infty} \int_{0}^{n} \frac{\left|\Delta^{2} f(x, t)\right|^{n}}{2^{n} t^{n+1}} d t=\int_{0}^{n}\left(\sum_{n=2}^{\infty} \frac{\left|\Delta^{2} f(x, t)\right|^{n}}{2^{n} t^{n+1}}\right) d t<\infty .
$$

The last integral is the same as the integral (1).

Conversely, assume that the integral (1) is finite for almost every $x$. This implies that $\epsilon_{x}(t)<1$ for almost all $t \in\left(0, \eta_{x}\right)$. Thus $\phi\left(\epsilon_{x}(t)\right)<1$ for almost all $t \in\left(0, \eta_{x}\right)$, and hence

$$
\int_{0}^{\eta} \frac{\tilde{\epsilon}_{x}^{2}(t)}{t} d t \leqq \int_{0}^{\eta} \frac{\underset{\epsilon_{x}}{2}(t)}{t \phi\left(\epsilon_{x}(t)\right)} d t, \quad \eta=\eta_{x} .
$$

By $\left[8\right.$, p. 253] $f$ has a derivative in $L_{2}$ at almost all points of $E$, and hence $f_{a p}^{\prime}(x)$ exists a. e. in $E$ (for an explicit statement of this see e.g. [5]). Since $\Delta^{2} f(x, t)=O(t), t \in N_{x},\left|N_{x}\right|=0$, we have by Lemma 3 that $f_{*}^{\prime}(x)$ exists a. e. in $E$. Application of Lemma 1 completes the proof. 
Using the same type of argument one can prove the following theorem.

Theorem 2. A measurable function $f: I_{0} \rightarrow R$ is equivalent to one which is differentiable a. e. on a measurable set $E \subset I_{0}$ if and only if for almost every $x \in E$ there is $\eta=\eta_{x}>0$ such that the sequence (2) is bounded.

Proof. The necessity is due to Marcinkiewicz [2]. For the sufficiency simply observe that the boundedness of (2) at a certain $x$ implies that $\left|\Delta^{2} f(x, t)\right| \leqq M t, 0<t<\eta$, except for $t$ belonging to a certain set $N_{x}$ of measure zero, where $M>1$ is the bound of the sequence (2). As above, the finiteness of the first term of the sequence (2) implies the existence of $f_{a p}^{\prime}(x)$ a. e. in $E$, and hence the Lemmas 3 and 1 complete the proof.

5. The Theorem 2 raises the natural question whether or not the boundedness of the sequence (2) can be relaxed to either (i) the lim sup as $p \rightarrow \infty$ is finite or (ii) each term in the sequence is finite. We will show that the answer is negative in both cases.

(i) Let $\sigma(t)=(\log 1 / t)^{-1 / 2}$, and consider the lacunary trigonometric series

$$
f(x)=\sum \sigma\left(\frac{1}{2^{n}}\right) \frac{\sin 2^{n} x}{2^{n}} .
$$

By $[8$, p. 250] the function $f(x)$ is almost nowhere differentiable and satisfies $\Delta^{2} f(x, t)=O(t \sigma(t))$ for each $x$. From $[8$, p. 252] we infer that

$$
\int_{0}^{1} \frac{\left|\Delta^{2} f(x, t)\right|^{2}}{t^{3}} d t=\infty
$$

for almost all $x$ and any $\eta>0$. However, for $n>2$ and $\eta$ small,

$$
\int_{0}^{\eta} \frac{\left|\Delta^{2} f(x, t)\right|^{n}}{t^{n+1}} d t \leqq M \int_{0}^{n} \frac{\sigma^{n}(t)}{t} d t \leqq M \int_{0}^{n} \frac{\sigma^{8}(t)}{t} d t<\infty,
$$

where $M$ is some constant.

(ii) Let $\tau(x)=2^{-1 / x}, x>0$, and let $A=U[1 / n, 1 / n+\tau(1 / 2 n)]$. Then $A$ is a union of disjoint closed intervals such that $|A \cap J|=o(\tau(|J|))$ as $|J| \rightarrow 0,0 \in J$, where $J$ is the notation for an interval. We need the following lemma.

LEMMA 4. Let $C$ be closed and nowhere dense in $I_{0}$, and let $G_{i}=\left(a_{i}, b_{i}\right)$, $i=1,2, \cdots$, be its complementary intervals. Then there exists $A \subset \cup G_{i}$ such that (i) $A$ is a union of disjoint closed intervals, (ii) $C \subset \bar{A}-A$, 
where $\bar{A}$ is the closure of $A$, (iii) $|A \cap J|=o(\tau(|J|))$, as $J \rightarrow x, x \in J$, for any $x \in C$.

Proof. Let $A_{i} \subset G_{i}$ be the set constructed relative to $a_{i}$ the same way as $A$ was constructed above relative to 0 . There exists $\delta_{i}>0$ such that $|J| \leqq \delta_{i}, a_{i} \in J$, implies $\left|A_{i} \cap J\right| \leqq\left(1 / 2^{i}\right) \tau(|J|)$. We may also suppose that $\delta_{i}<\left(1 / 2^{i}\right) \tau\left(b_{i}-a_{i}-\delta_{i}\right)$. Let $A_{i}^{*}=A_{i} \cap\left[a_{i}, a_{i}+\delta_{i}\right]$, and let $A=\bigcup A_{i}^{*}$. An easy computation establishes (iii) (see e.g. [5]).

We are now ready to prove the following theorem.

THEOREM 3. Let $C$ be a closed nowhere dense subset of $I_{0}$ of positive measure. Then there exists a measurable function $f: I_{0} \rightarrow R$ such that

(i) $\int_{0}^{1}\left(\left|\Delta^{2} f(x, t)\right|^{n} / t^{n+1}\right) d t<\infty, n=2,3, \cdots$, for almost all $x \in C$, and

(ii) if $g$ is equivalent with $f$, then $g$ is not differentiable at any point of $C$.

Proof. Let $A \subset I_{0}-C$ be the set constructed in Lemma 4, and let $f$ be the characteristic function of $A$. It is clear that (ii) is satisfied. Using [8, p. 280], we only need to show that for $x \in C$,

$$
\left\{\frac{1}{2 h} \int_{-h}^{h}|f(x+t)|^{n} d t\right\}^{1 / n}=o(h), \quad n=2,3, \cdots,
$$

i.e., $f$ has a $L_{n}$-derivative, which is zero, at each $x \in C$. Let $x \in C$, and let $A_{0}=\{a-x: a \in A\}$. Then

$$
\int_{-h}^{h}|f(x+t)|^{n} d t=\left|A_{0} \cap[-h, h]\right|=o(\tau(2 h)),
$$

and so is $o\left(h^{n+1}\right)$.

\section{BIBLIOGRAPHY}

1. H. Blumberg, The measurable boundaries of an arbitrary function, Acta Math. 65 (1935), 263-282.

2. J. Marcinkiewicz, Sur quelques intégrales du type de Dini, Ann. Soc. Polon. Math. 17 (1936), 42-50.

3. - Sur les séries de Fourier, Fund. Math. 27 (1936), 38-69.

4. C. J. Neugebauer, Symmetric and smooth functions of several variables, Math. Ann. 153 (1964), 285-292.

5. - Smoothness and differentiability in $L_{p}$, Studia Math. 25 (1964), 81-91.

6. S. Saks, Theory of the integral, Warszawa-Lwow, 1937.

7. E. M. Stein and A. Zygmund, Smoothness and differentiability of functions, Ann. Univ. Sci. Budapest, Sectio Math. 3-4 (1960-1961), 295-307.

8. - On the differentiability of functions, Studia Math. 23 (1964), 247-283.

9. A. Zygmund, Trigonometric series, 2 vols., 2nd. ed., Cambridge Univ. Press, Cambridge, 1959.

Purdue University 